\title{
PanViz 2.0: AI-driven Visual Analytics to Adapt to the Novel Challenges of COVID-19
}

\author{
Luke S. Snyder Jieqiong Zhao Audrey Reinert \\ University of Oklahoma Arizona State University University of Oklahoma

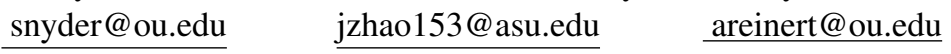 \\ Guizhen Wang \\ Purdue University \\ wang1908@purdue.edu \\ David S. Ebert \\ University of Oklahoma \\ ebert@ou.edu
}

\begin{abstract}
The ongoing and evolving COVID-19 pandemic has resulted in tremendous negative effects on people's daily lives. It is critical for decision makers such as health care officials and governors to foresee potential impacts and make timely decisions. We present PanViz 2.0, a visual analytics application that combines an epidemic model and AI-driven analytics to infer the best-fit parameters to enable the adaptation to ongoing pandemics at multiple spatial aggregations (nation wide, state level, and county level). Our experiments for predicting the fatality cases in each county of the state of Oklahoma demonstrate the flexibility of our application in adapting to various scenarios and regions.
\end{abstract}

\section{Introduction}

Decision makers need to make effective and timely decisions to respond to the evolving threats posed by a pandemic. Often, these decision makers are called upon to make highly consequential decisions using data collected from a variety of geographically and temporally disparate sources - ranging from hospital capacity to social media posts - within a relatively short-time frame. However, the size, complexity, and scope of the data presented to these decision makers make it difficult to assess the short-, medium-, and long-term consequences of a decision without substantial analytical support. Decision support systems, especially visual analytic systems, can help an individual make informed decisions during pandemic conditions.

Traditionally, epidemic models have been applied to predict the percent of a population that will be affected by influenza. Specifically, multiple parameters such as global infection rate, mortality rate, hospitalization rate, the speed of transmission (measured by miles per day for a person-to-person contact model) contribute to the final prediction. Decision-makers, such as health care officials, can provide some parameters based on their domain knowledge and observations of the ongoing pandemic to improve prediction accuracy and efficacy. Therefore, it is critical to use an interactive visual analytics application to assist the exploratory investigation and refinement of what-if scenarios in order to provide improved mitigation.

We adopt an automatic approach to speed up the parameter investigation process since fine-tuning a set of parameters simultaneously is tedious and time-consuming. The integration of AI-based optimization approaches in an interactive visual analytics environment can expedite the discovery of best-fit parameters in various scenarios for different pandemics and a range of spatial aggregates (e.g., nation wide, state level, and county level). Furthermore, the parameters recommended by the AI approach have specific physical meanings in an epidemic model. Decision-makers can inspect the recommended parameters and decide if they match the ongoing situation and gain further insights by setting the parameters to preferred ranges that most effectively represent the evolving situation.

In response to the COVID-19 global pandemic, we developed an AI-integrated application based upon the original PanViz, a visual analytics application for supporting pandemic preparedness through a tightly-coupled epidemiological model and interactive interface [1], to accommodate the new patterns and dynamics of COVID-19 and infer best-fit model parameters. In this paper, we summarize our contributions as the following:

- We develop an interactive web application that incorporates epidemiological modeling and temporal and spatial visualizations

- We incorporate AI inference techniques to recommend optimal parameters for improved modeling flexibility and predictions of different pandemic scenarios and spatial ranges

- We provide an example of COVID-19 model fitting for Oklahoma, a southern state in the USA 


\section{Background}

The spread of Ebola in the US during 2014 demonstrated how detecting and responding to a disease early are key steps to avoiding and mitigating the short-, medium-, and long-term effects of pandemics on population health $[2,3]$. However, effective detection and response to a pandemic are nontrivial, requiring the use of accurate information, advanced analytics, and predictions to carefully plan various interdiction/mitigation strategies. The recent COVID-19 pandemic has demonstrated excellent progress in collecting useful, timely pandemic data throughout the world, including novel sources such as social mobility data and public health electronic surveillance data, to assess and prevent the disease spread. While these data sources can greatly improve situational awareness, facilitate effective mitigation response, and provide feedback on current public health policies, they are often incomplete and biased. Further, the size and complexity of these data sources create difficulties, as decision-makers may not be able to appropriately compare and assess the outcome of different interdiction strategies without computational support.

One method to provide this computational support to decision makers comes from the development of visual analytics systems. VA tools and techniques allow decision-makers to address complex problems that require tightly-coupled human and machine analysis to develop effective solutions. In the context of pandemic response, decision-makers can utilize VA systems to efficiently compare differences (e.g., nearand long-term effects) between various intervention methods, such as the effect that mask wearing will have on disease spread.

Pandemics occur on a geospatial scale that can be difficult for individuals to fully comprehend. On an intuitive level, people understand that regions with smaller, distal populations will experience the first recorded case later than large population centers near the origin of the outbreak. While this heuristic understanding is largely correct, a county health official cannot reliably predict when a disease will appear nor inform the public of steps to take to prevent further spread using naive models.

VA systems, such as PanViz, can be designed to clearly communicate complicated and nuanced findings from statistical epidemiological models to a larger audience. For example, the conventional deterministic Susceptible, Infectious, Recovered (SIR) epidemiological model is widely adopted to estimate the number of infected people in a closed population. PanViz [1] introduced a visual analytics application to assist interactive exploration of what-if scenarios, such that users can dynamically refine the parameters of an epidemic model in order to obtain the number of infected, deceased, and recovered individuals. Besides developing an epidemic model based on SIR, PanViz offers the ability to take preventive measures (e.g., school closure, social distancing) to reduce the disease spread. Additionally, spatial (choropleth map) and temporal (line charts) visualizations are utilized in PanViz to illustrate the geographical distribution of infected population at the county level. The effectiveness of PanViz has been verified by health care officials. However, it is still challenging to find out the best-fit parameters since PanViz uses pre-defined parameters to generate the prediction results. It is tedious and repetitive to fine-tune multiple parameters simultaneously. In this paper, we build upon the epidemic model used in PanViz and automatically recommend parameters to adapt to COVID-19 scenarios.

\section{Related Work}

In this section, we organize literature into three categories: decision support systems, COVID-19 modeling, and visual analytics for COVID-19.

\subsection{Decision Support Systems}

One of the primary concerns that arises when using epidemiological models to map the spread of a disease such as COVID-19 is the inherent complexity of how these models function. A decision maker, or more generally a member of the public, may intuitively understand the relationship between population density and the rate at which a disease spreads within a community. These intuitive, heuristic models are beneficial when making decisions under duress. The psychology underpinning the benefit of heuristics stems from the fact that simplification supports humans in coping with limited information processing capacities. However, hueristics can be easily biased by what data is currently available and how it is presented $[4,5]$.

AI enabled decision support systems have been proposed as a solution to enable humans to make better decisions when faced with uncertainty. The primary benefits of using AI include the ability to discern complex patterns and relationships in large volumes of data. AI enabled decision support systems have been successfully used in clinical diagnostics [6, 7], operational efficiency [8], inventory management [9], and military decision making [10]. Any discussion of AI enabled decision support systems would be incomplete without mentioning the inherent limitations 
of AI in decision making. These limitations include: a lack of transparency in how the system is assessing different outcomes and the potential to reinforce or obscure preexisting biases or trends in the data [11, 12]. Therefore, we adopted epidemic models that have physical meanings to inform the interpretability of the underlying model. However, the exact contributions of a set of model parameters are still obfuscated, as it is difficult to understand their impacts in isolation. Therefore, we employ an optimization approach to infer the best-fit parameters from observed COVID-19 data.

\subsection{COVID-19 Modeling}

The surge of the COVID-19 pandemic has drawn high attention in academia. Before the COVID-19 outbreak, respiratory disease research accumulated sufficient experiences, e.g., modeling the reproduction number factor in the virus transmission [13]. After the outbreak, significant preliminary research has been conducted in a timely manner to investigate COVID-19 related issues. Punn et al. [14] utilized traditional machine learning approaches to forecast confirmed/death/recovered cases worldwide for the following ten days. Yang et al. [15] modeled the impact of intervention control policies (e.g., travel limit, quarantine of epidemic areas) on mitigation of disease containment. Their model assumed that these policies could affect population migration dynamics, a key factor affecting propagation of COVID-19. Their results revealed that a five-day delay of intervention policy implementation in China would have increased infected cases three-fold. In light of overwhelming hospital service demands, researchers at the Institute of Health Metrics and Evaluation (IHME) [16] built statistical models to predict COVID-19 related death rates and hospital service needs on a daily basis. The predictions ran at the spatial scale of European countries or USA states. Policy makers can use these predictions to enact certain rules to control and reduce disease spread. However, these works do not integrate visual interpretability for their models.

\subsection{Visual Analytics for COVID-19}

There is a body of academic literature that demonstrates the value of visual analytics in disease control and prevention. Araz et al. [17] reported that pandemic response exercises simulated in a visual analytic environment could increase readiness of healthcare decision-makers for epidemiological outbreaks. Malik et al. [18] presented a visual analytic approach to interactively explore temporal trends of multiple diseases. PanViz [1] is a visual analytic system for healthcare departments to interactively simulate their disease control strategies for an influenza pandemic in terms of outbreak origins and transmission rates. Afzal et al. [19] developed an interactive tool for public health officials and researchers to interactively explore the impact of their decision points on the future course of disease spread and mitigation. After the outbreak of COVID-19, visual analytics has been immediately applied to analyze relevant data. Dey et al. [20] conducted the first attempt on COVID-19 data analysis by combining multiple data sources into a visual analytics environment. Currently, the COVID-19 trend is evolving rapidly, and the visual analytics community is urgently needed to incorporate human observed knowledge to investigate and mitigate disease contamination. Our work is on this track, as we developed a flexible and adaptive visual analytics environment to predict the trend of COVID-19.

\section{PanViz 2.0}

Determination of optimal disease modelings involves multiple analytical procedures such as parameter settings and model comparisons. Visual analytics can build effective analytic environments for end users to interactively explore these procedures and choose appropriate models [21]. To facilitate such exploratory analysis, we develop the PanViz 2.0 visual analytic framework, a re-engineered version of PanViz [1, 22, 19] informed by our experiences in developing and deploying visual analytic decision support systems over the past 10 years.

PanViz was initially designed for use as a desktop application, limiting its portablity, scalablity, and flexibility. PanViz 2.0 builds on the initial visual design of PanViz while converting the software into an intuitive web-based application (Fig. 1, 2). This conversion addresses the portability and scalability problems outlined earlier, as several users can access the system from any compatible web browser. Users can adjust simulation and model parameters (Fig. 1 left panel), interactively visualize the geographic spread of the pandemic (Fig. 1 center panel), and visualize countyand state-level case data (Fig. 1 right panel).

PanViz 2.0 also provides interactive exploration and analysis of different mitigation strategies. Specifically, users can place decision points, such as school closures (Fig. 3), along any part of a trend line to visualize the effects on infection, death, and hospitalization counts. Users can control the precise impacts of a given decision measure by adjusting its infection reduction probability. This is particularly useful in cases where a decision measure's exact impact may not be known a priori 


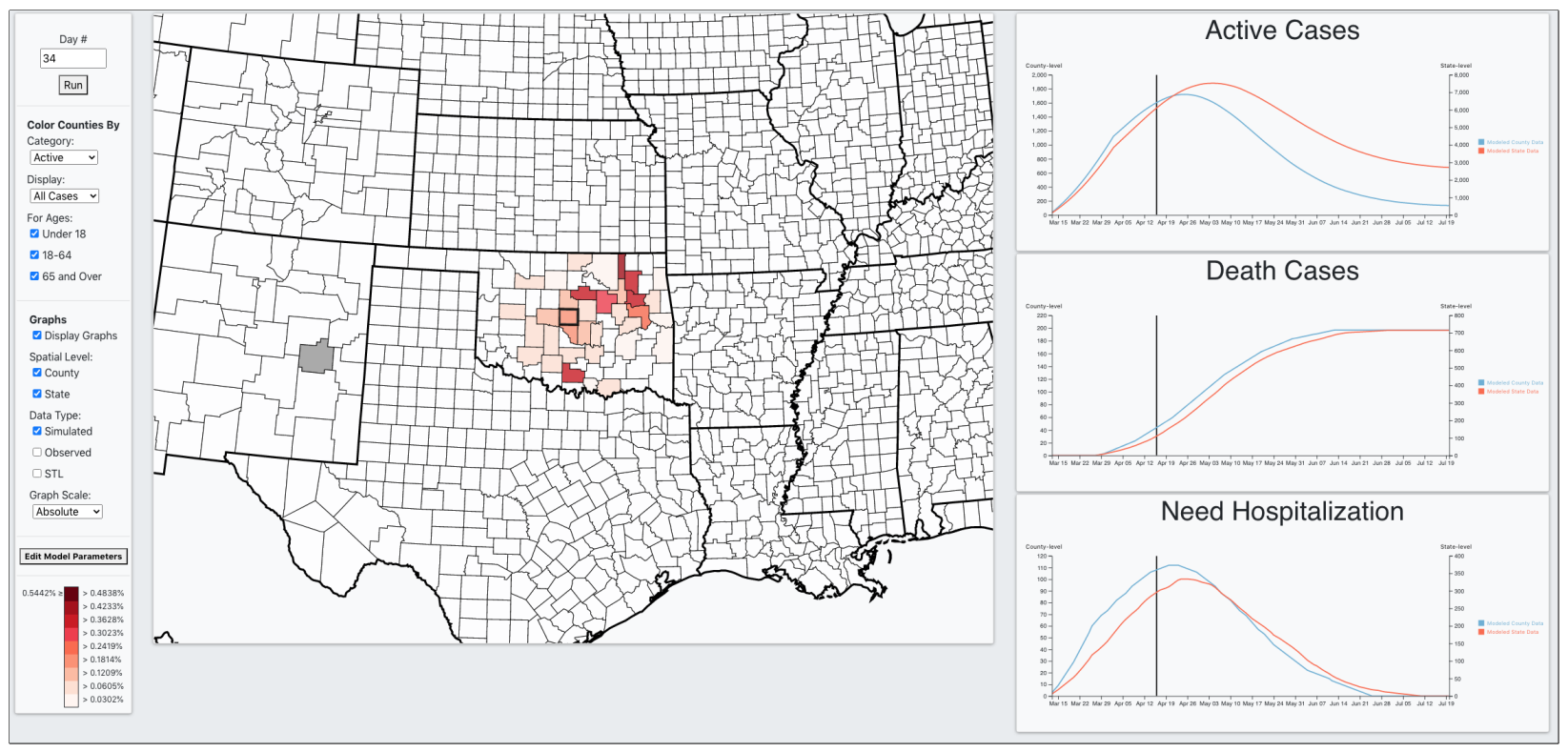

Figure 1. Overview of PanViz 2.0: (left) control panel to configure model parameters and simulation settings, (middle) geographic distribution of cases, (right) temporal visualization of infections, fatalities, and hospitalizations, the light red line indicates the prediction for state level (with corresponding axis on the right side) and the light blue line indicates the prediction for a selected county (with corresponding axis on the left side).

(such as in novel situations like COVID-19) and should therefore be experimented with (and updated as new data are collected) to assess how effective it may or may not be at different stages of the disease.

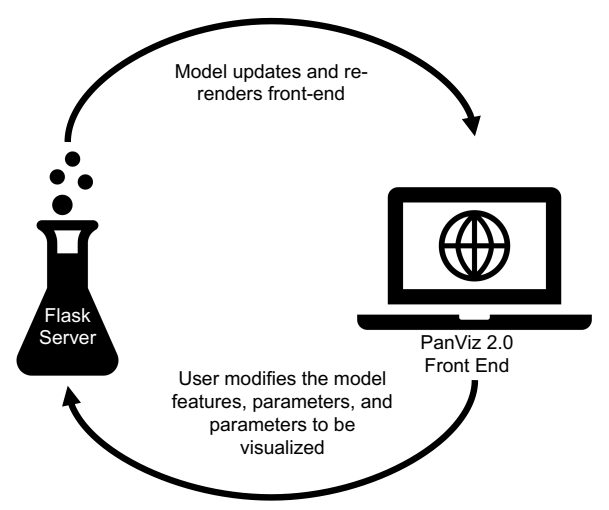

Figure 2. The architecture of PanViz 2.0.

\subsection{Base Epidemiological Model}

PanViz employs a mathematical epidemiological model based on the work of Malone et al. [23], which uses a system of non-linear difference equations derived from traditional epidemic models to calculate county-level disease dynamics. PanViz also integrates airport transportation spread dynamics for improved modeling. A PanViz 2.0 user can supply county level population data, including demographics and density, the observed mortality and recovery rate of the disease, hospitalization rate, and baseline and modified disease prevalence (Fig. 4). Disease prevalence is defined as the percentage of the entire national population that would be infected with the disease if no action is taken. The user can also stipulate a custom origin point of the disease or select a major airport as an origin point. For a detailed explanation the mechanics of the model, please see $[23,24]$.

\section{AI-driven Parameter Inference}

In this section, we discuss our AI-driven approach for inferring model parameters to simulate the COVID-19 pandemic, as well as initial results to demonstrate its effectiveness.

\subsection{Approach}

To accurately simulate the COVID-19 pandemic, we combine PanViz's base epidemiological model with machine learning to infer model parameters (e.g., speed of transmission, mortality rate, etc.). This allows us to maintain the strong mathematical principles underlying pandemic dynamics, while also optimizing the model for different situations (e.g., different state 


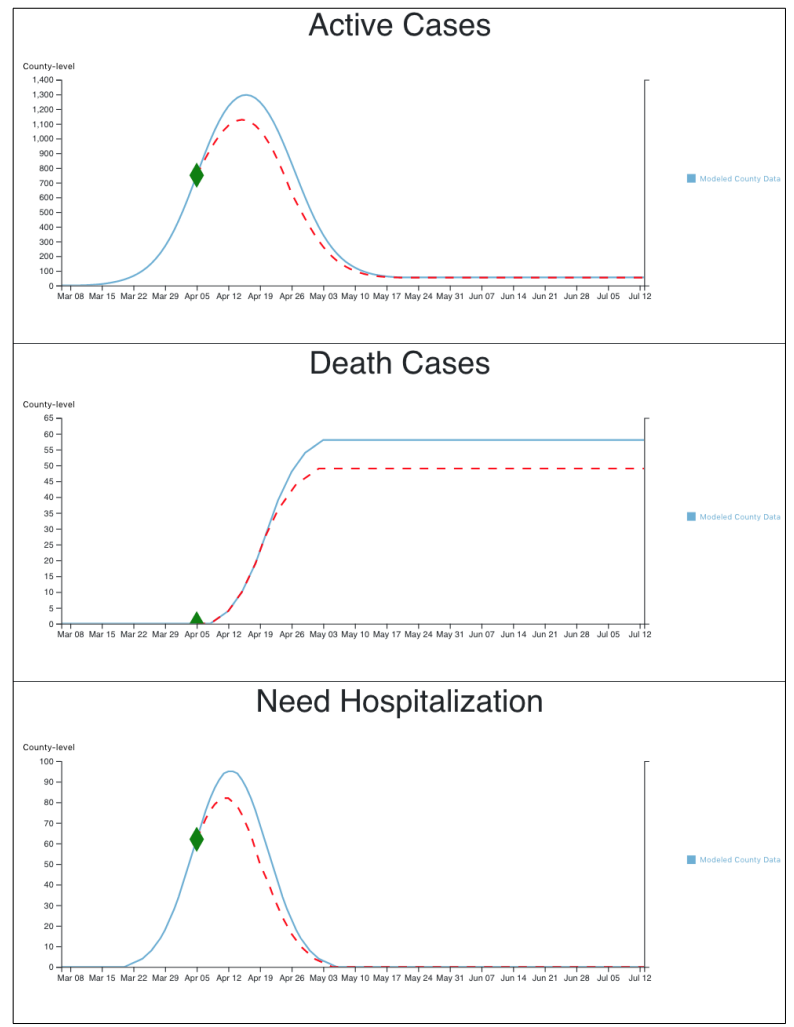

Figure 3. Users can place decision points along any trend line to view their effects on infections, deaths, and hospitalizations. The green decision point in this figure corresponds to school closures with an assumed infection reduction of $25 \%$.

or county data). Similar to the approach used by covid19-projections.com, we utilize a simple but effective machine learning technique called grid search to identify the optimal parameters. Grid search iterates over all possible parameter combinations (within reasonable intervals as informed by previous research) and computes the loss for each combination by comparing the model's simulated data against observed data. We use the Root Mean Square Error (RMSE) in Equation 1 to compute the deviation between prediction and observation across the pandemic time range, where $y_{i}$ corresponds to the reported value (ground truth) on day $i, \hat{y}_{i}$ corresponds to the predicted value on day $i$, and $N$ is the total number of days. The optimal model parameters are derived by minimizing the RMSE:

$$
R M S E=\sqrt{\frac{\sum_{i=1}^{N}\left(y_{i}-\hat{y}_{i}\right)^{2}}{N}}
$$

The specific details of the grid search algorithm used to minimize RMSE are shown in Algorithm 1.

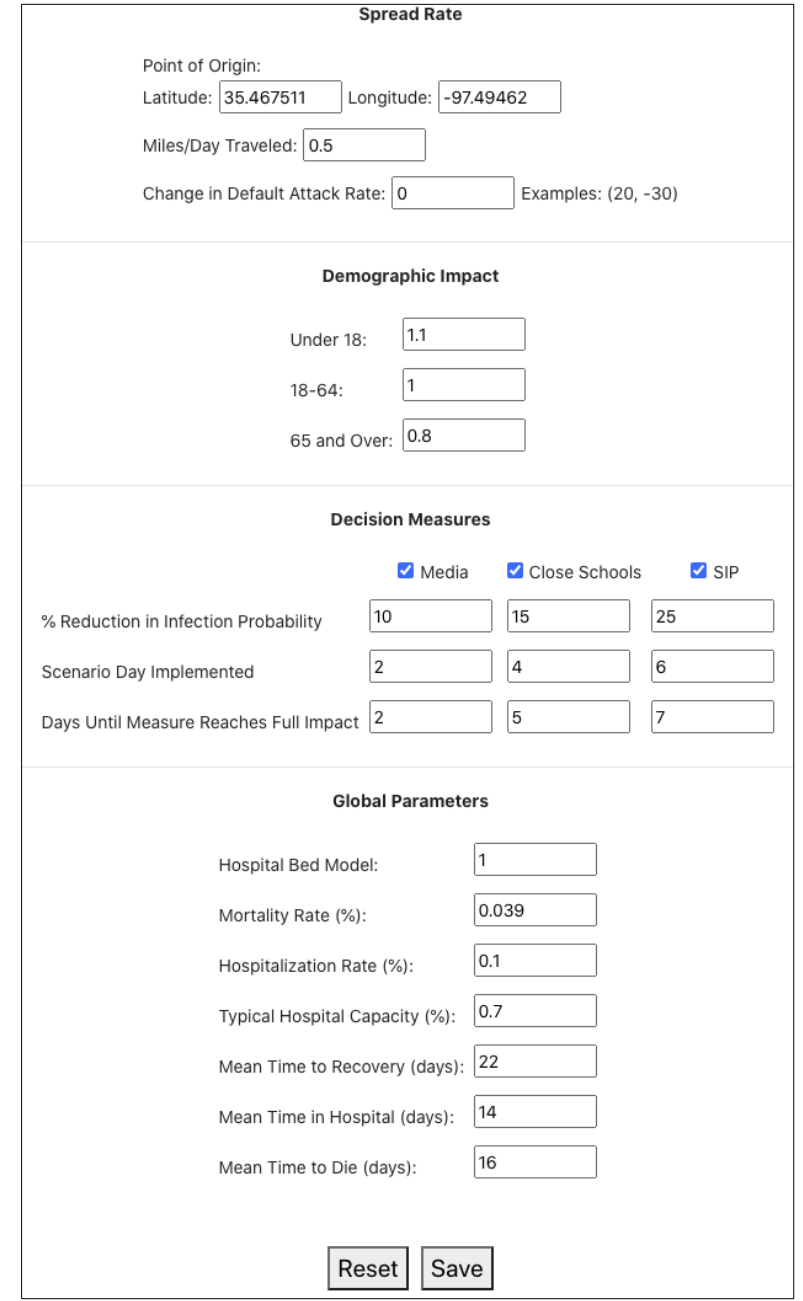

Figure 4. User-configurable epidemic model parameters.

To assess the effectiveness of our approach, we focus on Oklahoma state COVID-19 data released by The New York Times ${ }^{1}$. The data contains the cumulative number of infections and deaths for each county in Oklahoma over a 130-day period from March 1, 2020 to July 8, 2020 , with gaps on days where information was not reported. We concentrate our analysis on fatalities, as the infection numbers vary among different data sources due to unreliable testing and asymptomatic infection, although extending our approach to any data source is straightforward.

We execute grid search (with the fatality data) for each county in Oklahoma to determine the associated optimal parameters. We infer values for parameters such as spread rate speed, peak amplitude, mortality rate, and decision measure impacts since they may fluctuate

\footnotetext{
${ }^{1}$ github.com/nytimes/covid-19-data
} 


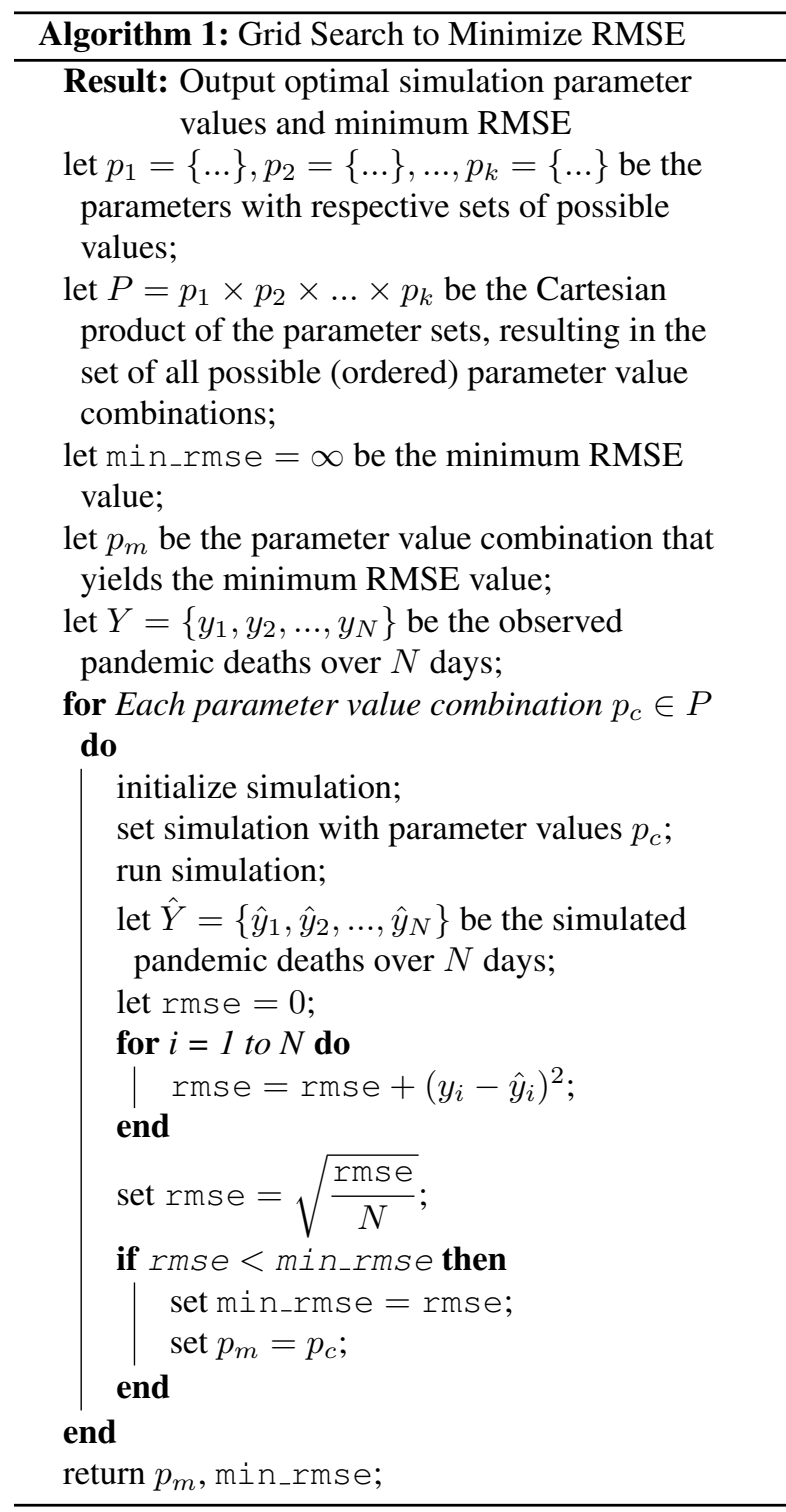

between different counties. We use fixed values (as informed by research) for parameters which are unlikely to change between regions, such as the mean time to recover, mean time to die, and demographic (age) impacts.

\subsection{Results}

Fig. 5 illustrates the predicted (cumulative) fatality cases compared to the observed cases in Oklahoma County, OK, after parameter inference with grid search, which yielded a minimum RMSE value of 2.48. The dark and light blue lines in the center panel of Fig. 5 correspond to observed and predicted COVID-19 fatality data, respectively. The inferred parameters, as shown in Table 1, are a mortality rate of $3.2 \%$, amplitude (determines peak case number) of 0.0004 , peak day (determines time of maximum peak case number) of 25 , spread rate speed of 0.5 miles/day, school closure infection reduction of $15 \%$, media infection reduction of $10 \%$, and shelter-in-place infection reduction of $25 \%$.

\begin{tabular}{lr}
\hline Parameter & Inferred Value \\
\hline Mortality Rate & $3.2 \%$ \\
Amplitude & 0.0004 \\
Peak Day & 25 \\
Spread Rate Speed & $0.5 \mathrm{mi} / \mathrm{day}$ \\
School Closure Infection Reduction & $15 \%$ \\
Media Infection Reduction & $10 \%$ \\
Shelter-in-place Infection Reduction & $25 \%$ \\
\hline
\end{tabular}

Table 1. Inferred optimal parameters for Oklahoma County, OK.

Parameter inference allows decision-makers to make predictions regarding important future trends, as the visuals in Fig. 5 also provide the predicted fatality numbers after the observed data ends on day 130 . Specifically, the number of predicted deaths plateau near the end, indicating that future observed COVID-19 total fatalities may also stabilize. It is important to note that the optimal parameters may naturally fluctuate as more observed data is collected in the future, although we expect the model to still predict reasonably well. We plan to introduce confidence intervals in the future to account for uncertainty.

Of practical importance is that although the optimal parameters are derived from the fatality data, they can be used to infer the infection and hospitalization counts, as shown in the top and bottom panels of Fig. 5. This can be useful since, as previously mentioned, the observed infection and hospitalization data may be incorrect and misleading due to asymptomatic infection and unreliable testing.

To assess the reliability and sensitivity of our parameter inference approach (grid search), we construct our own test sets since the "ground truth" parameters are not known (due to the novelty and ongoing research of COVID-19) and, therefore, we cannot directly compare the optimal parameters inferred from grid search. We generate 50 different test sets by adding random noise from the normal distribution $\mathcal{N}(\mu=0, \sigma=1)$ to the daily death cases of the best-fit model (Table 1). We then execute grid search on each test set to determine how well it can identify the original best-fit model parameters from the noise. The result statistics of all test sets for Oklahoma 
County, OK, are shown in Table 2. Specifically, the averages and standard deviations for the mortality rate, amplitude, peak day, spread rate speed, school closure infection reduction, media infection reduction, and shelter-in-place infection reduction are 3.376 $\pm 0.434 \%, 3.760 \times 10^{-4} \pm 4.833 \times 10^{-5}, 24.6 \pm 2.828$, $0.5 \pm 0 \mathrm{mi} /$ day, $15 \pm 0 \%, 10 \pm 0 \%$, and $25 \pm 0 \%$. Each of the average values are close to (i.e., $\pm 1 \sigma$ ) the original best-fit parameters in Table 1 ("ground truth" parameters in this case), with some values remaining the exact same (e.g., spread rate speed, school closure infection reduction, media infection reduction, and shelter-in-place infection reduction). This indicates that our grid search approach is fairly effective and reliable at identifying the optimal parameters from noisy data and, therefore, should provide a close estimate to the true COVID-19 parameters.

\begin{tabular}{|c|c|}
\hline Parameter & $\begin{array}{l}\text { Average Value } \\
( \pm 1 \sigma)\end{array}$ \\
\hline Mortality Rate & $3.376 \pm 0.434 \%$ \\
\hline Amplitude & $\begin{array}{l}3.760 \times 10^{-4} \pm \\
4.833 \times 10^{-5}\end{array}$ \\
\hline Peak Day & $24.6 \pm 2.828$ \\
\hline Spread Rate Speed & $0.5 \pm 0 \mathrm{mi} / \mathrm{day}$ \\
\hline School Closure Infection Reduction & $15 \pm 0 \%$ \\
\hline Media Infection Reduction & $10 \pm 0 \%$ \\
\hline Shelter-in-place Infection Reduction & $25 \pm 0 \%$ \\
\hline
\end{tabular}

Table 2. Average and standard deviation of inferred optimal parameters from $\mathbf{5 0}$ generated (noisy) test sets of daily deaths counts in Oklahoma County, OK.

It is important to note that estimating the optimal parameters from collected COVID-19 data is challenging due to the imprecision in the data. Specifically, the data collection methods and standards may vary between counties and states and potentially contain human reporting errors. While this is one of our primary reasons for focusing on fatality cases (since they may be more straightforward to report and less likely to miscalculate), we plan to investigate soft computing methods such as Support Vector Machines and Artificial Neural Networks which can be better equipped to handle data imprecision and uncertainty [25].

\section{Discussion}

While traditional models are capable of accurately simulating the spread of diseases such as Ebola or Influenza, the accuracy of these models at predicting

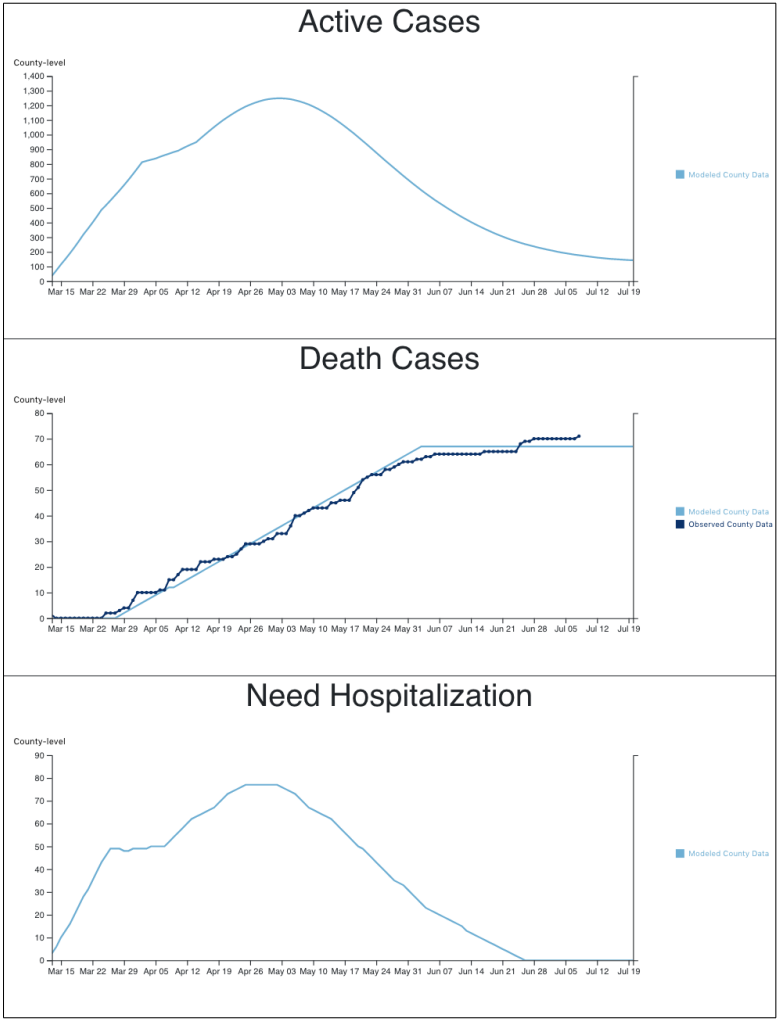

Figure 5. Active, death, and hospitalization cases for Oklahoma County, OK, with optimized parameters. The observed COVID-19 data is colored in dark blue.

the spread of novel diseases is potentially limited. During the early stages of a pandemic, public health officials may not have a sufficient understanding of how a disease is transmitted, what the symptoms of the disease are, and which population demographics are at increased risk. We know from current epidemiology studies that COVID-19 spreads from person to person via respiratory droplets [26, 27], but there is ongoing research to determine how long it takes for an individual exposed to COVID-19 to be infected, when an infected individual can spread the virus, and the effect of asymptomatic spread [28]. Unraveling this problem is complicated in regions with low population densities, counties with limited case histories, areas with unique characteristics that impact the propagation of COVID-19 such as a small town with a meatpacking plant [29, 30, 20], and Native American Reservations [31]. Due to geographic and economic factors, these areas are the least prepared for an onslaught of COVID-19 cases [32, 20]. Further, hospitals and pharmacies serving low population density areas need to evaluate how best to utilize their limited budgets and available resources to meet the upcoming demand. But, access to reliable information to support the decision 
making process is poor $[33,34]$.

To account for these uncertainties, PanViz 2.0 ingests collected data to facilitate data-driven comparisons against model simulations. Further, our AI-driven parameter inference allows users to interactively analyze the optimized model for future predictions, as well as continue exploring and tuning the model settings, which can improve situational awareness and decision-making during pandemic and public health crises.

Decision measures such as mandated masking wearing or social distancing are critical for impeding the spread of respiratory diseases like COVID-19 [35, 36]. However, these measures can be deployed over differing time scales, making direct comparisons of effectiveness difficult. PanViz 2.0 allows users to answer questions about the effectiveness of different intervention regimes by visually comparing their effects on virus spread and case numbers.

\section{Conclusion and Future Work}

As recent news events have shown, the pressures facing public health officials are immense and life-altering for thousands of individuals. Even a decision as seemingly small as when to announce a particular policy can save the lives of tens of thousands of individuals. Making these complicated decisions without computational support can be very difficult. Through the continued development and refinement of PanViz 2.0, we aim to provide decision-makers with a tool to help them synthesize and visualize a wide variety of data types - ranging from hospital capacity to Facebook posts - for evaluating the outcomes of various decisions to further their goal of making life saving choices.

\subsection{Future Work}

While real-life data can be useful for tuning epidemiological models, there is still a degree of uncertainty regarding future trends. Therefore, we plan to introduce sophisticated techniques for exploration of what-if scenarios and interdiction planning by allowing decision-makers to visually compare different parameter combinations and their effects on disease dynamics. For instance, users may be interested in assessing how the disease spread changes with different combinations of hospital capacities, rural and urban density impacts, and decision measures. Our previous work on reducing anomaly false positives while modeling incident occurrence for pandemic preparedness [24] will also be integrated to provide trustable predictive analytics.
PanViz 2.0 currently supports AI-driven parameter inference without human guidance. We plan to incorporate interactive machine learning for user-driven parameter learning to improve predictions and transparency. As an example, users could visualize the grid search parameter space to investigate reasons for differences in RMSE and predictions. Further, we plan to investigate alternative AI modeling approaches. For instance, multiple probabilistic SEIR model simulations with different parameters could be used to train machine learning classifiers such as neural networks to predict the appropriate parameters. Decision-makers could then continue to tune and adapt the trained model through interactive analysis.

We infer model parameters that remain static over the entire pandemic simulation, although it is likely that some parameters may vary temporally with disease spread and interdiction planning phases. We plan to accommodate this by learning optimal parameter sets for each temporal phase. For instance, the mortality rate and disease spread may decrease after certain public policies are implemented, such as school closures and mask wearing.

\section{References}

[1] R. Maciejewski, P. Livengood, S. Rudolph, T. F. Collins, D. S. Ebert, R. T. Brigantic, C. D. Corley, G. A. Muller, and S. W. Sanders, "A pandemic influenza modeling and visualization tool," Journal of Visual Languages \& Computing, vol. 22, pp. 268 - 278, Aug 2011.

[2] B. Kelly, L. Squiers, C. Bann, A. Stine, H. Hansen, and M. Lynch, "Perceptions and plans for prevention of Ebola: results from a national survey," BMC Public Health, vol. 15, no. 1, p. 1136, 2015.

[3] T. Salaam-Blyther, "The 2014 ebola outbreak: International and U.S. responses," Current Politics and Economics of Africa, vol. 7, no. 4, pp. 523-557, 2014.

[4] S. Luan, J. Reb, and G. Gigerenzer, "Ecological rationality: Fast-and-frugal heuristics for managerial decision making under uncertainty," Academy of Management Journal, vol. 62, no. 6, pp. 1735-1759, 2019.

[5] M. Raue and S. G. Scholl, "The use of heuristics in decision making under risk and uncertainty," in Psychological perspectives on risk and risk analysis, pp. 153-179, Springer, 2018.

[6] Q. Yang, A. Steinfeld, and J. Zimmerman, "Unremarkable AI: Fitting intelligent decision support into critical, clinical decision-making processes," in Proceedings of the 2019 CHI Conference on Human Factors in Computing Systems, pp. 1-11, 2019.

[7] A. Rodríguez-Ruiz, E. Krupinski, J.-J. Mordang, K. Schilling, S. H. Heywang-Köbrunner, I. Sechopoulos, and R. M. Mann, "Detection of breast cancer with mammography: effect of an artificial intelligence support system," Radiology, vol. 290, no. 2, pp. 305-314, 2019. 
[8] E. B. Beşikçi, O. Arslan, O. Turan, and A. I. Ölçer, "An artificial neural network based decision support system for energy efficient ship operations," Computers \& Operations Research, vol. 66, pp. 393-401, 2016.

[9] P. Kumar, Development of Demand Forecast and Inventory Management Decision Support System Using AI Techniques. $\mathrm{PhD}$ thesis, National Institute of Technology Karnataka, Surathkal, 2018.

[10] K. van den Bosch and A. Bronkhorst, "Human-AI cooperation to benefit military decision making," in Proceedings of the NATO IST-160 Specialist' meeting on Big Data and Artificial Intelligence for Military Decision Making, NATO, June 2018.

[11] C. Xu, S. M. Brown, and C. Grant, "Detecting simpson's paradox," in Proceedings of the Thirty-First International Flairs Conference, May 2018.

[12] F. Tramer, V. Atlidakis, R. Geambasu, D. Hsu, J.-P. Hubaux, M. Humbert, A. Juels, and H. Lin, "FairTest: Discovering unwarranted associations in data-driven applications," in Proceedings of the IEEE European Symposium on Security and Privacy (EuroS\&P), pp. 401-416, IEEE, 2017.

[13] H. L. Tessmer, K. Ito, and R. Omori, "Can machines learn respiratory virus epidemiology?: A comparative study of likelihood-free methods for the estimation of epidemiological dynamics," Frontiers in Microbiology, vol. 9 , p. 343,2018

[14] N. S. Punn, S. K. Sonbhadra, and S. Agarwal, "Covid-19 epidemic analysis using machine learning and deep learning algorithms," medRxiv, June 2020.

[15] Z. Yang, Z. Zeng, K. Wang, S. S. Wong, W. Liang, M. Zanin, P. Liu, X. Cao, Z. Gao, Z. Mai, J. Liang, X. Liu, S. Li, Y. Li, F. Ye, W. Guan, Y. Yang, F. Li, S. Luo, Y. Xie, B. Liu, Z. Wang, S. Zhang, Y. Wang, N. Zhong, and J. He, "Modified SEIR and AI prediction of the epidemics trend of COVID-19 in China under public health interventions," Journal of Thoracic Disease, vol. 12, pp. 165-174, Mar 2020.

[16] IHME COVID-19 Health Service Utilization Forecasting Team and C. J. Murray, "Forecasting the impact of the first wave of the COVID-19 pandemic on hospital demand and deaths for the USA and European Economic Area countries," tech. rep., Apr 2020.

[17] O. M. Araz, M. Jehn, T. Lant, and J. W. Fowler, "A new method of exercising pandemic preparedness through an interactive simulation and visualization," Journal of medical systems, vol. 36, no. 3, pp. 1475-1483, 2012.

[18] A. Malik, S. Afzal, E. Hodgess, D. S. Ebert, and R. Maciejewski, "VACCINATED — visual analytics for characterizing a pandemic spread vast 2010 mini challenge 2 award: Support for future detection," in Proceedings of the IEEE Symposium on Visual Analytics Science and Technology, pp. 281-282, Oct 2010.

[19] S. Afzal, R. Maciejewski, and D. S. Ebert, "Visual analytics decision support environment for epidemic modeling and response evaluation," in Proceedings of the IEEE Symposium on Visual Analytics Science and Technology, pp. 191-200, Dec 2011.

[20] S. K. Dey, M. M. Rahman, U. R. Siddiqi, and A. Howlader, "Analyzing the epidemiological outbreak of COVID-19: A visual exploratory data analysis approach," Journal of Medical Virology, vol. 92, pp. 632-638, Apr 2020.
[21] D. A. Keim, J. Kohlhammer, F. Mansmann, T. May, and F. Wanner, Mastering the Information Age: Solving Problems with Visual Analytics, ch. 2.2, pp. 10-11. Eurographics Association, 2010.

[22] R. Maciejewski, S. Rudolph, S. J. Grannis, and D. S. Ebert, "The day-of-the-week effect: a study across the indiana public health emergency surveillance system," in Proceedings of International Society for Disease Surveillance Annual Conference Advances in Disease Surveillance, vol. 5, p. 44, 2008.

[23] J. D. Malone, R. Brigantic, G. A. Muller, A. Gadgil, W. Delp, B. H. McMahon, R. Lee, J. Kulesz, and F. M. Mihelic, "Us airport entry screening in response to pandemic influenza: modeling and analysis," Travel Medicine and Infectious Disease, vol. 7, no. 4, pp. 181-191, 2009.

[24] R. Maciejewski, S. Afzal, A. J. Fairfield, A. Ghafoor, D. S. Ebert, N. Ayyaz, and M. Ahmed, "Enabling syndromic surveillance in pakistan," Online Journal of Public Health Informatics, vol. 5, Mar 2013.

[25] S. Mitra, S. K. Pal, and P. Mitra, "Data mining in soft computing framework: a survey," IEEE transactions on neural networks, vol. 13, no. 1, pp. 3-14, 2002.

[26] S. Chaudhuri, S. Basu, P. Kabi, V. R. Unni, and A. Saha, "Modeling the role of respiratory droplets in covid-19 type pandemics," Physics of Fluids, vol. 32, no. 6, p. 063309, 2020.

[27] L. Bourouiba, "Turbulent gas clouds and respiratory pathogen emissions: potential implications for reducing transmission of covid-19," Jama, vol. 323, no. 18, pp. 1837-1838, 2020.

[28] X. Yu and R. Yang, "Covid-19 transmission through asymptomatic carriers is a challenge to containment," Influenza and Other Respiratory Viruses, 2020.

[29] J. E. Hobbs, "Food supply chains during the covid-19 pandemic," Canadian Journal of Agricultural Economics/Revue canadienne d'agroeconomie, 2020.

[30] J. Middleton, R. Reintjes, and H. Lopes, "Meat plants-a new front line in the covid-19 pandemic," BMJ : British Medical Journal, p. m2716, July 2020.

[31] M. Kakol, D. Upson, and A. Sood, "Susceptibility of southwestern american indian tribes to coronavirus disease 2019 (covid-19)," The Journal of Rural Health, 2020.

[32] A. N. Roy, J. Jose, A. Sunil, N. Gautam, D. Nathalia, and A. Suresh, "Prediction and spread visualization of COVID-19 pandemic using machine learning," Preprints, May 2020.

[33] G. Adunlin, P. Z. Murphy, and M. Manis, "Covid-19: How can rural community pharmacies respond to the outbreak?," The Journal of Rural Health, 2020.

[34] J. E. Fried, D. T. Liebers, and E. T. Roberts, "Sustaining rural hospitals after covid-19: The case for global budgets," JAMA, 2020.

[35] J. Howard, A. Huang, Z. Li, Z. Tufekci, V. Zdimal, H.-M. van der Westhuizen, A. von Delft, A. Price, L. Fridman, L.-H. Tang, et al., "Face masks against covid-19: an evidence review," Preprints 2020040203, July 2020.

[36] W. Lyu and G. L. Wehby, "Community use of face masks and covid-19: Evidence from a natural experiment of state mandates in the us: Study examines impact on covid-19 growth rates associated with state government mandates requiring face mask use in public.," Health Affairs, pp. 10-1377, 2020. 